\title{
Transarterial chemoembolization with raltitrexed-based or floxuridine-based chemotherapy for unresectable colorectal cancer liver metastasis
}

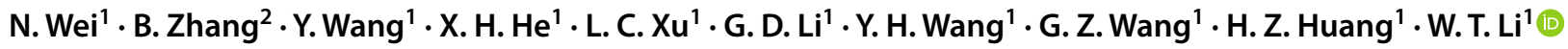

Received: 29 June 2018 / Accepted: 2 September 2018 / Published online: 10 October 2018

(c) The Author(s) 2018

\begin{abstract}
Purpose To evaluate and compare the efficiency and safety of raltitrexed- or floxuridine (FUDR)-based transarterial chemoembolization (TACE) in patients with unresectable colorectal cancer liver metastasis (CRCLM).

Methods We conducted a retrospective analysis of 81 patients with unresectable CRCLM who failed systemic chemotherapy and were treated with TACE in our department from Oct 2014 to Oct 2017. Of these, 61 patients received TACE using raltitrexed, oxaliplatin, and pirarubicin (raltitrexed group), and 20 received TACE using FUDR, oxaliplatin, and pirarubicin (FUDR group). The objective response rate (ORR), disease control rate (DCR), overall survival (OS, from the first TACE), progression-free survival (PFS, from the first TACE), and adverse reactions were evaluated and compared between the two groups, and prognostic factors for OS were analyzed.

Results The ORRs of the raltitrexed group and FUDR group were 67.2 and $45.0 \%$, respectively $(P=0.076)$, and the DCRs were 86.9 and $80.0 \%$, respectively $(P=0.452)$. The median OS (from first TACE) was 14.0 months in the raltitrexed group and 13.0 months in the FUDR group $(P=0.556)$. The median PFS (from first TACE) was 2.1 months in the raltitrexed group and 2.4 months in the FUDR group $(P=0.878)$. Univariate and multivariate analyses showed that the primary tumor site, Child-Pugh class, and combination with local ablation (RFA or CRA) were independent significant factors affecting survival. There were no significant differences in adverse reactions between the two groups $(P>0.05)$, and no treatmentrelated death occurred in either group.

Conclusion TACE treatment based on raltitrexed or FUDR is an efficient and safe alternative choice for treating unresectable CRCLM.
\end{abstract}

Keywords Colorectal cancer $\cdot$ Liver metastasis $\cdot$ Raltitrexed $\cdot$ Floxuridine $\cdot$ Transcatheter arterial chemoembolization

\section{Introduction}

Colorectal cancer (CRC) is one of the most common malignancies and is the third leading cause of cancer-related death in the western world [1]. In China, CRC is the fifth

N. Wei, B. Zhang and Y. Wang have contributed to this work equally (joint first authors).

W. T. Li

liwentao98@126.com

1 Department of Interventional Radiology, Fudan University Shanghai Cancer Center, Shanghai 200032, China

2 Department of Interventional Medical, Peoples Hospital Xintai City, Taishan Medical University, Xintai 271200, China leading cause of cancer death, with approximately 376,300 estimated new cases and 191,000 deaths in 2015, and the incidence rate is rising [2]. More than $50 \%$ of CRC patients develop liver metastasis, which is one of the main causes of death for CRC patients [3]. Liver resection may be the only chance of long-term survival for patients with unresectable CRC liver metastasis (CRCLM) [4]. However, more than $80 \%$ of CRCLM patients are not suitable for liver surgery, because the remaining liver function would be insufficient if the metastatic tumor was to be removed completely [3, 5]. Systemic chemotherapy, including chemotherapy regimens of FOLFOX, FOLFIRI, CAPEOX, and FOLFOXIRI, is the main treatment option for metastatic CRC [6]. The survival benefit has been considerably improved by the combination of chemotherapy with targeted therapy, with a median overall survival (OS) of up to 30 months [7-9]. 
Nevertheless, the survival rate is inferior for patients with refractory metastatic CRC who fail two lines of systemic chemotherapy, with a median OS time of 7.1-8.8 months [10]. If the previous chemotherapy has failed, transarterial chemoembolization (TACE) is considered as an alternative option for CRCLM. TACE has been demonstrated to have a higher tumor response rate and lower adverse effects than systemic chemotherapy [11, 12].

TACE is a therapeutic approach that delivers chemotherapeutic drugs and embolizing agents to the hepatic artery. Some chemotherapy drugs, such as mitomycin C, doxorubicin, cisplatin, and gemcitabine, are commonly used in TACE for CRCLM [13-15]. 5-Fluorouracil (5-FU) is a commonly used systemic chemotherapy drug in advanced CRC and is also used as one of the transarterial chemotherapy drugs in TACE for the treatment of CRCLM [16]. Floxuridine (FUDR), a derivative of 5-FU, is usually used in hepatic arterial infusion (HAI) because of its high hepatic extraction rate and low toxicity $[17,18]$. Some early clinical studies have reported that the use of FUDR in HAI produces median response rates of $40-45 \%$ and a median survival time of 17 months for metastatic CRC [19, 20].

Raltitrexed is a specific thymidylate synthase inhibitor. It has the same target as FUDR, but their mechanisms of action are different [21]. Raltitrexed has been widely used for the treatment of metastatic CRC, alone or in combination with oxaliplatin [22-24]. The combination of raltitrexed and oxaliplatin shows response rates of $41-54 \%$ and median survival times of 14.6-15.6 months for metastatic CRC [25-28]. Several previous clinical studies have shown that raltitrexed- and 5-FU-based chemotherapy has similar efficacies in the treatment of advanced CRC $[25,26]$. However, a few clinical studies concerning the evaluation of the efficiency and safety of raltitrexed- or FUDR-based TACE for the treatment of CRCLM have been reported. The purpose of this retrospective study was to evaluate and compare the efficiency and safety of raltitrexed- (raltitrexed, oxaliplatin, and pirarubicin) or floxuridine-based (floxuridine, oxaliplatin, and pirarubicin) TACE in patients with unresectable CRCLM who failed two lines of systemic chemotherapy.

\section{Materials and methods}

\section{Patient population}

This retrospective study was approved by the Ethics Committee of Fudan University Cancer Center. Between Oct 2014 and Oct 2017, 81 unresectable CRCLM cases that received TACE using raltitrexed, oxaliplatin, and pirarubicin (raltitrexed group, $n=61$ ) or floxuridine, oxaliplatin, and pirarubicin (FUDR group, $n=20$ ) were included. The inclusion criteria for TACE were as follows: a histologically confirmed diagnosis of CRC and complete resection of the primary tumor; inoperable liver metastases and contraindications to liver resection; the failure of two lines of systemic chemotherapy or discontinuation of chemotherapy due to significant side effects; an Eastern Cooperative Oncology Group (ECOG) performance status of 0 or 1; tumor involvement of less than $70 \%$ of the liver volume and adequate liver or renal dysfunction (total bilirubin serum levels $<3 \mathrm{mg} /$ $\mathrm{dL}$, serum albumin level $>20 \mathrm{~g} / \mathrm{L}$, serum creatinine level $<2 \mathrm{mg} / \mathrm{dL}$ ). For all patients, the treatment for liver metastases with systemic chemotherapy was stopped before 4 weeks of TACE therapy. Patients who had extrahepatic metastases were included if their main lesion remained in the liver. Patients were excluded if they had inadequate medical records or received TACE therapy not based on raltitrexed (raltitrexed, oxaliplatin, and pirarubicin) or floxuridine (floxuridine, oxaliplatin, and pirarubicin).

\section{TACE procedure}

After local anesthesia, the Seldinger technique was used to puncture the femoral arterial. The size, number, position, and blood supply of the liver lesions were determined by the introduction of the 5F-RH catheter (Boston Scientific, USA) into the abdominal aorta and celiac trunk by digital subtraction angiography (DSA). Then, a 3F microcatheter (Terumo Corp, Tokyo, Japan) was used for superselective intubation into the hepatic artery and tumor-nourishing vessels. The dosage of drugs and lipiodol (Laboratoire Andre Guerbet, France) depended on the condition of the tumor and blood vessels. In both groups, raltitrexed (4 mg, Tai-Tianqing Pharmaceutical, Co., Ltd., Nanjing, China) or FUDR (1.0 g, Nantong Jinghua, Pharmaceutical, Co., Ltd., Nantong, China) was perfused through the catheter first. Subsequently, oxaliplatin (100 mg, Sanofi Synthelabo France, Paris, France) and pirarubicin $(60 \mathrm{mg}$, Wan Le Pharmaceutical, Shen Zhen, Co., Ltd., Shen Zhen, China) were mixed with lipiodol $(10 \mathrm{ml})$, and the tumor was embolized with this mixture. Finally, once again, DSA radiography was used to determine the effect of TACE therapy. If a hepatic arteriovenous fistula was found during the operation, appropriately sized gelatin sponge particles or PVA could be used to block the arteriovenous fistula. After TACE, antiemetic, hepatoprotection, and antacid drugs were used routinely.

TACE was repeated every 4-6 weeks. When the tumor response was evaluated as partial response (PR), stable disease (SD) or progressive disease (PD) and no serious complications and contraindications occurred, TACE was performed again. If the tumor did not progress, the frequency of TACE was reduced appropriately. The criteria for terminating TACE therapy included an ECOG performance status 
$>2$, Child-Pugh $\mathrm{C}$ liver function, or an evaluation of tumor response showing complete response (CR).

\section{Response assessment}

For all patients, the upper abdominal enhanced computed tomography (CT) or magnetic resonance imaging (MRI) was reviewed after 1 month of TACE therapy and then every month until death or loss to follow-up. The objective response rate (ORR) and disease control rate (DCR) were evaluated using Response Evaluation Criteria in Solid Tumor (RECIST) version 1.1. OS was calculated from the date of TACE therapy to the date of the last follow-up or death. Progression-free survival (PFS) was calculated from the start date of TACE therapy to the date of disease progression or death. The adverse reactions were assessed according to the National Cancer Institute-Common Terminology Criteria for Adverse Events (NCI-CTACE) version 4.0. The major adverse reactions were classified as levels $0-4$. The last follow-up time of this study was Mar 30, 2018.

\section{Statistical analysis}

The statistical analysis was performed using SPSS version 20.0 (SPSS Inc. Chicago, IL, USA). GraphPad Prism 6 (GraphPad software, Inc., La Jolla, CA, USA) was used to generate charts. Continuous variables are shown as the mean \pm standard deviation (SD). Qualitative data are expressed as the frequency and percentage. Statistical analyses were performed using $t$ tests for measurement data, and Chi-square tests or Fisher's exact tests for count data. The survival analysis for OS and PFS was estimated with the Kaplan-Meier methods, and the significance of OS and PFS between the two groups was determined using the log-rank test. The clinical variables included sex, age at first TACE, primary tumor site, tumor pathological grade, time to liver metastasis, tumor number, maximum tumor diameter, intrahepatic vascular invasion, Child-Pugh class, combination with radiofrequency ablation (RFA) or cryoablation (CRA) treatment, and extrahepatic metastasis. Univariate analysis was applied to all variables, and any variable with a $P$ value $<0.10$ in the univariate analysis was entered into the multivariate analysis. Cox model was used to analyze the prognostic factors related to survival. $P<0.05$ was considered to indicate a statistically significant difference.

\section{Results}

\section{Patient characteristics}

A total of 81 patients who received TACE were enrolled. There were 61 patients in the raltitrexed group and 20 patients in the FUDR group. The baseline characteristics of the patients are summarized in Table 1. No significant differences were found in terms of sex, age at first TACE, number of TACE cycles, primary tumor site, tumor pathological grade, time to liver metastasis, tumor number, maximum tumor diameter, intrahepatic vascular invasion, Child-Pugh class, additional RFA or CRA after TACE, or extrahepatic metastasis between the two groups $(P>0.05)$. Patients in the raltitrexed group received a median of three TACE cycles, and those in the FUDR group received a median of three TACE cycles. Thirty-three patients $(40.7 \%)$ received additional local treatment including RFA or CRA after TACE.

\section{Tumor response}

Table 2 shows the results of tumor response evaluated according to RECIST 1.1 for two groups. Among the 61 patients in the raltitrexed group, eight $(13.1 \%)$ CRs, thirtythree (54.1\%) PRs, twelve (19.7\%) SDs, and eight (13.1\%) PDs were observed. The ORR and DCR were $67.2 \%$ and $86.9 \%$, respectively. Among the 20 patients in the FUDR group, two (10\%) CRs, seven (35\%) PRs, seven (35\%) SDs, and four (20\%) PDs were observed. The ORR and DCR were 45.0 and $80.0 \%$, respectively. Although the ORR was higher in the raltitrexed group than in the FUDR group, there was no statistical significance between the two groups in terms of the ORR $(P=0.076)$, or DCR $(P=0.452)$.

\section{Survival}

The median follow-up time was 20 months. At the end of the follow-up period, $11(18.0 \%)$ patients in the raltitrexed group and 3 (15.0\%) patients in the FUDR group were still alive. A Kaplan-Meier survival analysis showed no significant differences between the two groups in terms of OS and PFS. The median OS (from first TACE) was 14.0 months in the raltitrexed group and 13.0 months in the FUDR group $(P=0.556)$ (Fig. 1). The median PFS (from first TACE) was 2.1 months in the raltitrexed group and 2.4 months in the FUDR group, respectively ( $P=0.878)$ (Fig. 2). The Cox univariate and multivariate analysis identified several prognostic factors of OS (Table 3). The univariate analysis demonstrated that primary tumor site, tumor number, maximum tumor diameter, intrahepatic vascular invasion, Child-Pugh class, and the combination with RFA or CRA displayed a significant association with OS. Of these, primary tumor site (HR $=0.425,95 \%$ CI 0.192-0.940, $P=0.035)$, Child-Pugh class $(\mathrm{HR}=0.401,95 \% \mathrm{CI} 0.198-0.812, P=0.011)$, and the combination with RFA or CRA (HR $=0.463,95 \%$ CI $0.231-0.887, P=0.021$ ) were further confirmed by multivariate analysis to be independent factors for OS after TACE. 
Table 1 Basic clinical characteristics of the two groups

\begin{tabular}{|c|c|c|c|c|}
\hline & $\begin{array}{l}\text { Overall cohort } \\
(n=81)\end{array}$ & $\begin{array}{l}\text { Raltitrexed } \\
\text { group }(n=61)\end{array}$ & FUDR group $(n=20)$ & $P$ value \\
\hline Sex & & & & 0.855 \\
\hline Male & 54 & 41 & 13 & \\
\hline Female & 27 & 20 & 7 & \\
\hline Age (years) at first TACE & $61.1 \pm 8.7$ & $60.6 \pm 8.9$ & $62.5 \pm 8.2$ & 0.255 \\
\hline Number of TACE cycles & $3.9 \pm 3.7$ & $4.0 \pm 4.1$ & $3.4 \pm 1.9$ & 0.175 \\
\hline Primary tumor site & & & & 0.674 \\
\hline Right hemicolon & 19 & 15 & 4 & \\
\hline Left hemicolon & 62 & 46 & 16 & \\
\hline Tumor pathological grade & & & & 0.115 \\
\hline Poor & 25 & 16 & 9 & \\
\hline Well or intermediate & 56 & 45 & 11 & \\
\hline Time to liver metastasis & & & & 0.331 \\
\hline Synchronous (<6 months) & 48 & 38 & 10 & \\
\hline Metachronous (> 6 months) & 33 & 23 & 10 & \\
\hline Tumor number & & & & 0.452 \\
\hline Single & 12 & 8 & 4 & \\
\hline Multiple & 69 & 53 & 16 & \\
\hline Maximum tumor diameter & & & & 0.651 \\
\hline$\leq 5 \mathrm{~cm}$ & 40 & 31 & 9 & \\
\hline$>5 \mathrm{~cm}$ & 41 & 30 & 11 & \\
\hline Intrahepatic vascular invasion & & & & 0.538 \\
\hline Presence & 16 & 13 & 3 & \\
\hline Absence & 65 & 48 & 17 & \\
\hline Child-Pugh class & & & & 0.185 \\
\hline A & 65 & 51 & 14 & \\
\hline $\mathrm{B}$ & 16 & 10 & 6 & \\
\hline Combined with RFA or CRA & & & & 0.547 \\
\hline Yes & 33 & 26 & 7 & \\
\hline No & 48 & 35 & 13 & \\
\hline Extrahepatic metastasis & & & & 0.731 \\
\hline Presence & 18 & 13 & 5 & \\
\hline Absence & 63 & 48 & 15 & \\
\hline
\end{tabular}

Table 2 Comparison of response between the raltitrexed group and FUDR group

\begin{tabular}{lllll}
\hline Outcome & Group & & $\chi^{2}$ & $P$ value \\
\cline { 2 - 3 } & $\begin{array}{l}\text { Raltitrexed group } \\
(n=61)\end{array}$ & FUDR group $(n=20)$ & & \\
\hline Complete response (CR) & 8 & 2 & & \\
Partial response (PR) & 33 & 7 & & \\
Stable disease (SD) & 12 & 7 & & \\
Progressive disease (PD) & 8 & 4 & 3.146 & 0.076 \\
Objective response rate (ORR) & $41(67.2 \%)$ & $9(45.0 \%)$ & 0.266 & 0.452 \\
Disease control rate (DCR) & $53(86.9 \%)$ & $16(80.0 \%)$ & &
\end{tabular}

\section{Adverse reactions}

Table 4 summarizes the most common adverse reactions in this study. The most common treatment-related adverse reactions included hematological, liver, and kidney function; gastrointestinal symptoms; other reactions (non-infectious fever, abdominal pain and neuropathy), and no significant differences were found between the raltitrexed group and 




Fig. 1 Kaplan-Meier curves of the overall survival (OS) for patients with unresectable CRCLM after TACE in the two groups

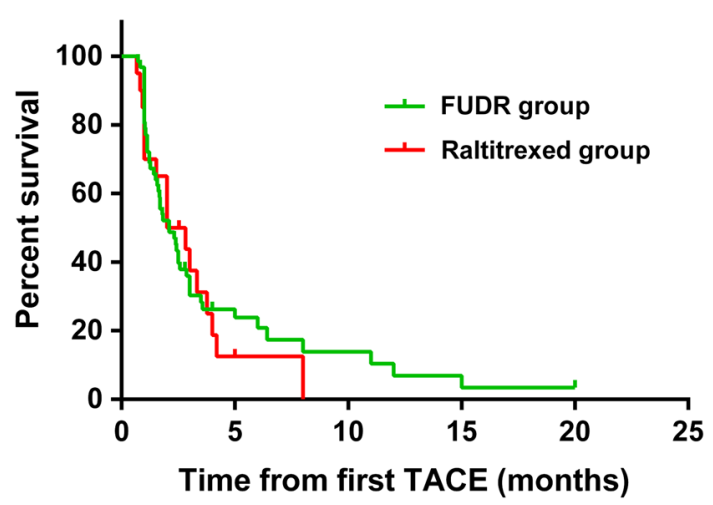

Fig. 2 Kaplan-Meier curves of the progression-free survival (PFS) for patients with unresectable CRCLM after TACE in the two groups

FUDR group $(P>0.05)$. No grade 3 or 4 hematological toxicity was observed in either group and no serious myelosuppression occurred. No treatment-related death occurred during the treatment period in either group.

\section{Discussion}

Systemic chemotherapy is the standard option for metastatic CRC. Local treatments, such as TACE and HAI, are alternative options for CRCLM with failed previous systemic chemotherapy. 5-FU and raltitrexed are commonly used as systemic chemotherapy drugs or applied in HAI for the treatment of metastatic CRC. A meta-analysis including 4622 patients with advanced CRC has shown that the OS and overall response rates are not significantly different between raltitrexed-based and fluorouracil-based chemotherapy [29]. Guo et al. evaluated the efficiency and safety of HAI chemotherapy using raltitrexed or 5-FU for CRCLM, and the result showed that there was no significant difference in survival between the two schemes [30].
FUDR is a derivative of 5-FU and is usually used in HAI for the treatment of CRCLM due to its high hepatic extraction rate and low toxicity $[17,18,31]$. Although the use of raltitrexed and FUDR in HAI has been reported, there are a few studies on the direct comparison of raltitrexedand FUDR-based regimens in TACE for the treatment of CRCLM.

Our study showed that the ORR and DCR of the raltitrexed group (raltitrexed, oxaliplatin, and pirarubicin) was higher than those of the FUDR group (floxuridine, oxaliplatin, and pirarubicin) (67.2 vs. $45.0 \%, 86.9$ vs. $80.0 \%$, respectively), but the differences were not statistically significant $(P>0.05)$. This result suggests that the raltitrexedbased regimen may be beneficial for the short-term control of liver lesions. The analysis of survival showed that the median PFS times (from first TACE) of the raltitrexed group and the FUDR group were 2.1 months and 2.4 months, respectively, with no statistically significant difference between them $(P=0.835)$. The median OS times (from first TACE) of the raltitrexed group and the FUDR group were 14.0 and 13.0 months, respectively, with no statistically significant difference between them $(P=0.556)$. The above results demonstrate that raltitrexed-based regimens can achieve similar effects to those FUDR-based regimens, which suggests that CRCLM patients may benefit from raltitrexed-based TACE therapy. Such results are similar to those of a comparison of raltitrexed with 5-FU in hepatic or systemic infusion chemotherapy $[29,30]$. Although there was no difference in OS between the two groups, the multivariate analysis showed that primary tumor site, Child-Pugh class, and the combination with local ablation (RFA or CRA) were independent predictors of OS. Our study indicated that TACE combined with other local treatments, such as RFA or CRA, may prolong the survival of CRCLM patients. The physiological basis of TACE or HAI is that a malignant tumor of the liver derives more than $80 \%$ of its blood supply from the hepatic arterial circulation [32]. After analyzing and comparing the DSA, we concluded that the blood supply characteristics of the metastatic tumor in the same liver were not identical and that a portion of the CRCLM patients would be resistant to chemotherapy, so not every lesion can be controlled well by TACE alone. To achieve a better control of liver metastases, local ablation combined with RFA and CRA is frequently used in our department, and the effectiveness and safety of RFA has been confirmed by some researchers $[33,34]$. In addition, the use of RFA or CRA is limited in lesions near major bile ducts and great vessels; therefore, the combination of two or more minimally invasive interventional methods to complement the other's advantages may be a necessary strategy.

Common adverse reactions were manifested as hematological, liver, and kidney function problems and digestive symptoms, and there was no significant difference between 
Table 3 Prognostic factors for overall survival

\begin{tabular}{|c|c|c|c|c|c|c|c|}
\hline \multirow[t]{2}{*}{ Factors } & \multirow[t]{2}{*}{$N$} & \multicolumn{3}{|c|}{ Univariate } & \multicolumn{3}{|c|}{ Multivariate } \\
\hline & & HR & $95 \% \mathrm{CI}$ & $P$ value & HR & $95 \% \mathrm{CI}$ & $P$ value \\
\hline Group & & 0.996 & $(0.572,1.732)$ & 0.988 & & & \\
\hline Raltitrexed group & 61 & & & & & & \\
\hline FUDR group & 20 & & & & & & \\
\hline Sex & & 1.263 & $(0.756,2.108)$ & 0.372 & & & \\
\hline Male & 54 & & & & & & \\
\hline Female & 27 & & & & & & \\
\hline Age (years) at first TACE & & 0.776 & $(0.475,1.267)$ & 0.311 & & & \\
\hline$\leq 60$ & 33 & & & & & & \\
\hline$>60$ & 48 & & & & & & \\
\hline Primary tumor site & & 0.400 & $(0.221,0.721)$ & 0.002 & 0.425 & $(0.192,0.940)$ & 0.035 \\
\hline Right hemicolon & 19 & & & & & & \\
\hline Left hemicolon & 62 & & & & & & \\
\hline Tumor pathological grade & & 1.116 & $(0.671,1.857)$ & 0.673 & & & \\
\hline Poor & 25 & & & & & & \\
\hline Well or intermediate & 56 & & & & & & \\
\hline Time to liver metastasis & & 1.095 & $(0.667,1.800)$ & 0.719 & & & \\
\hline Synchronous ( $<6$ months) & 48 & & & & & & \\
\hline Metachronous (> 6 months) & 33 & & & & & & \\
\hline Tumor number & & 0.477 & $(0.234,0971)$ & 0.041 & 0.740 & $(0.328,1.669)$ & 0.469 \\
\hline Single & 12 & & & & & & \\
\hline Multiple & 69 & & & & & & \\
\hline Maximum tumor diameter & & 1.623 & $(0.994,2.651)$ & 0.053 & 0.976 & $(0.468,2.038)$ & 0.949 \\
\hline$\leq 5 \mathrm{~cm}$ & 40 & & & & & & \\
\hline$>5 \mathrm{~cm}$ & 41 & & & & & & \\
\hline Intrahepatic vascular invasion & & 1.841 & $(1.045,3.243)$ & 0.035 & 1.232 & $(0.560,2.711)$ & 0.604 \\
\hline Presence & 16 & & & & & & \\
\hline Absence & 65 & & & & & & \\
\hline Child-Pugh class & & 0.370 & $(0.204,0.669)$ & 0.001 & 0.401 & $(0.198,0.812)$ & 0.011 \\
\hline A & 65 & & & & & & \\
\hline B & 16 & & & & & & \\
\hline Combined with RFA or CRA & & 0.463 & $(0.279,0.770)$ & 0.003 & 0.453 & $(0.231,0.887)$ & 0.021 \\
\hline Yes & 33 & & & & & & \\
\hline No & 48 & & & & & & \\
\hline Extrahepatic metastasis & & 0.835 & $(0.468,1.489)$ & 0.541 & & & \\
\hline Presence & 18 & & & & & & \\
\hline Absence & 63 & & & & & & \\
\hline
\end{tabular}

the two groups, indicating that CRCLM patients are tolerant of the two regimens. In this study, none of the patients had fatal myelosuppression, gastrointestinal bleeding, or hepatic abscess formation after TACE therapy. In general, the treatment of TACE is safe and reliable. Several studies have shown that the effect of TOMOX on liver function is more serious than that of FOLFOX, while cardiovascular toxicity is lighter [25, 35]. Therefore, for patients with normal liver function with high-risk factors for cardiovascular disease, the application of the TOMOX regimen may be safer. This study showed that all grade rising rates of postoperative transaminase were 78.7 and $50.0 \%$ in the raltitrexed group and FUDR group, respectively, and the all grade rising rates of total bilirubin after TACE were 70.5 and $30.0 \%$ in two groups, respectively. These results are similar to those of previous research. Although there was no statistical significance, these findings suggest that the damage to liver function caused by raltitrexed might be more common than that caused by FUDR. Therefore, liver protection should be actively pursued before TACE to improve patient tolerance. In addition, nausea and vomiting, abdominal pain, and non-infectious fever were common in both groups, and no statistically significant difference was observed. Considering that this series of 
Table 4 Adverse events of TACE therapy

\begin{tabular}{|c|c|c|c|c|c|c|}
\hline \multirow[t]{2}{*}{ Outcome } & \multicolumn{2}{|c|}{ Raltitrexed group $(n=61)$} & \multicolumn{2}{|c|}{ FUDR group $(n=20)$} & \multirow[t]{2}{*}{$\chi^{2}$} & \multirow[t]{2}{*}{$P$ value } \\
\hline & All grade & Grade 3-4 & All grade & Grade 3-4 & & \\
\hline \multicolumn{7}{|l|}{ Hematological } \\
\hline Leucopenia & 20 & 0 & 4 & 0 & 1.181 & 0.277 \\
\hline Thrombocytopenia & 16 & 0 & 5 & 0 & 0.012 & 0.913 \\
\hline Anemia & 28 & 0 & 9 & 0 & 0.005 & 0.944 \\
\hline \multicolumn{7}{|l|}{ Liver and kidney function } \\
\hline AST and ALT increased & 48 & 7 & 10 & 4 & 2.091 & 0.148 \\
\hline Total bilirubin increased & 43 & 6 & 6 & 2 & 0.927 & 0.336 \\
\hline Creatinine increased & 19 & 2 & 4 & 1 & 0.434 & 0.510 \\
\hline \multicolumn{7}{|l|}{ Gastrointestinal symptoms } \\
\hline Nausea/vomiting & 32 & 19 & 10 & 3 & 0.923 & 0.337 \\
\hline Diarrhea & 7 & 0 & 2 & 0 & 0.033 & 0.855 \\
\hline \multicolumn{7}{|l|}{ Other } \\
\hline Non-infectious fever & 34 & 3 & 13 & 1 & 0.013 & 0.909 \\
\hline Abdominal pain & 40 & 7 & 10 & 3 & 0.491 & 0.483 \\
\hline Neuropathy & 7 & 0 & 3 & 0 & 0.173 & 0.687 \\
\hline
\end{tabular}

symptoms is a typical embolization syndrome, these reactions may not be closely related to chemotherapy drugs, and there is a certain relationship between the type, usage, and dosage of an embolic agent and the tumor blood supply. After 2 or 3 days of treatment, the discomfort of most patients was relieved, and there were no complications such as intestinal obstruction, tumor rupture, or pulmonary embolism.

There were several limitations in the study. This study is a single-center retrospective study. The number of cases is relatively insufficient, and the results may be biased. In addition, long-term follow-up data were not available, and the curative effect could not be evaluated more comprehensively. Furthermore, this study revealed some problems to be solved. For example, the blood supply from some hepatic metastatic carcinomas is not rich in the TACE treatment, so there are inevitable differences between individual treatments. To ensure patient safety and strictly grasp the indications, TACE that is simultaneously combined with local treatment (RFA or CRA) may increase the curative effect, but further research is needed to verify this.

In conclusion, TACE treatment with either raltitrexed or FUDR is an efficient and safe alternative choice for treating unresectable CRCLM. Combined TACE and local ablation for treating unresectable CRCLM may be beneficial for survival, which deserves further study.

Funding This study was supported in part by National Key Research and Development Program of China (No. 2016YFC0106203) and Programme of Shanghai Hospital Development Center (No. SHDC22017102).

\section{Compliance with ethical standards}

Conflict of interest All authors declare no conflicts of interest for the present study.

Ethical approval This article does not contain any studies with human participants performed by any of the author.

Informed consent For this type of study formal consent is not required.

Open Access This article is distributed under the terms of the Creative Commons Attribution 4.0 International License (http://creativeco mmons.org/licenses/by/4.0/), which permits unrestricted use, distribution, and reproduction in any medium, provided you give appropriate credit to the original author(s) and the source, provide a link to the Creative Commons license, and indicate if changes were made.

\section{References}

1. Siegel RL, Miller KD, Jemal A. Cancer statistics 2018. CA Cancer J Clin. 2018;68(1):7-30.

2. Chen W, Zheng R, Baade PD, Zhang S, Zeng H, Bray F, et al. Cancer statistics in China, 2015. CA Cancer J Clin. 2016;66:115-32.

3. Leonard GD, Brenner B, Kemeny NE. Neoadjuvant chemotherapy before liver resection for patients with unresectable liver metastases from colorectal carcinoma. J Clin Oncol. 2005;23:2038-48.

4. Choti MA, Sitzmann JV, Tiburi MF, Sumetchotimetha W, Rangsin R, Schulick RD, et al. Trends in long-term survival following liver resection for hepatic colorectal metastases. Ann Surg. 2002;235:759-66.

5. Akgul O, Cetinkaya E, Ersoz S, Tez M. Role of surgery in colorectal cancer liver metastases. World J Gastroenterol. 2014;20:6113-22.

6. Fakih MG. Metastatic colorectal cancer: current state and future directions. J Clin Oncol. 2015;33(16):1809-24. 
7. Cremolini C, Loupakis F, Antoniotti C, Lupi C, Sensi E, Lonardi $\mathrm{S}$, et al. FOLFOXIRI plus bevacizumab versus FOLFIRI plus bevacizumab as first-line treatment of patients with metastatic colorectal cancer: updated overall survival and molecular subgroup analyses of the open-label, phase 3 TRIBE study. Lancet Oncol. 2015;16:1306-15.

8. Heinemann V, von Weikersthal LF, Decker T, Kiani A, VehlingKaiser U, Al-Batran SE, et al. FOLFIRI plus cetuximab versus FOLFIRI plus bevacizumab as first-line treatment for patients with metastatic colorectal cancer (FIRE-3): a randomised, openlabel, phase 3 trial. Lancet Oncol. 2014;15:1065-75.

9. Peeters M, Price TJ, Cervantes A, Sobrero AF, Ducreux M, Hotko $\mathrm{Y}$, et al. Randomized phase III study of panitumumab with fluorouracil, leucovorin, and irinotecan (FOLFIRI) compared with FOLFIRI alone as second-line treatment in patients with metastatic colorectal cancer. J Clin Oncol. 2010;28:4706-13.

10. Grothey A, Marshall JL, Seery TE. Current options for thirdline treatment of metastatic colorectal cancer. Clin Adv Hematol Oncol. 2016;14(3 Suppl 3):1-15.

11. Gruber-Rouh T, Marko C, Thalhammer A, Nour-Eldin NE, Langenbach M, Beeres M, et al. Current strategies in interventional oncology of colorectal liver metastases. Br J Radiol. 2016. https ://doi.org/10.1259/bjr.20151060.

12. Massmann A, Rodt T, Marquardt S, Seidel R, Thomas K, Wacker F, et al. Transarterial chemoembolization (TACE) for colorectal liver metastases - current status and critical review. Langenbecks Arch Surg. 2015;400(6):641-59.

13. Albert M, Kiefer MV, Sun W, Haller D, Fraker DL, Tuite CM, et al. Chemoembolization of colorectal liver metastases with cisplatin, doxorubicin, mitomycin C, ethiodol, and polyvinyl alcohol. Cancer. 2011;117:343-52.

14. Vogl TJ, Gruber T, Balzer JO, Eichler K, Hammerstingl R, Zangos S. Repeated transarterial chemoembolization in the treatment of liver metastases of colorectal cancer: prospective study. Radiology. 2009;250:281-9.

15. Ghanaati H, Mohammadzadeh V, Mohammadzadeh A, Firouznia K, Mohammadzadeh M, Motevali M, et al. Efficacy of transarterial chemoembolization on lesion reduction in colorectal liver metastases. Acta Med Iran. 2012;50:535-40.

16. Sanz-Altamira PM, Spence LD, Huberman MS, Posner MR, Steele G Jr, Perry LJ, et al. Selective chemoembolization in the management of hepatic metastases in refractory colorectal carcinoma: a phase II trial. Dis Colon Rectum. 1997;40:770-5.

17. Li C, Gu Y, Zhao M, Yuan Y, Wang F, Wang Z, et al. Phase I trial of hepatic arterial infusion (HAI) of floxuridine with modified oxaliplatin, 5-fluorouracil and leucovorin (m-FOLFOX6) in Chinese patients with unresectable liver metastases from colorectal cancer. Cancer Chemother Pharmacol. 2014;74:1079-87.

18. Ensminger WD. Intrahepatic arterial infusion of chemotherapy: pharmacologic principles. Semin Oncol. 2002;29:119-25.

19. Balch CM, Urist MM. Intraarterial chemotherapy for colorectal liver metastases and hepatomas using a totally implantable drug infusion pump. Recent Results Cancer Res. 1986;100:234-47.

20. Schwartz SI, Jones LS, McCune CS. Assessment of treatment of intrahepatic malignancies using chemotherapy via an implantable pump. Ann Surg. 1985;201:560-7.

21. Marsh S, McLeod HL. Thymidylate synthase pharmacogenetics in colorectal cancer. Clin Colorectal Cancer. 2002;1:179-80.
22. Feliu J, Castanon C, Salud A, Mel JR, Escudero P, Pelegrin A, et al. Phase II randomised trial of raltitrexed-oxaliplatin vs raltitrexed-irinotecan as first-line treatment in advanced colorectal cancer. Br J Cancer. 2005;93:1230-5.

23. Cocconi G, Cunningham D, Van Cutsem E, Francois E, Gustavsson B, van Hazel G, et al. Open, randomized, multicenter trial of raltitrexed versus fluorouracil plus high-dose leucovorin in patients with advanced colorectal cancer. Tomudex Colorectal Cancer Study Group. J Clin Oncol. 1998;16:2943-52.

24. Vyzula R, Kocakova I, Demlova R, Kiss I, Dusek L, Jarkovsky J. Raltitrexed plus oxaliplatin in the second-line treatment of metastatic colorectal cancer. Neoplasma. 2006;53:119-27.

25. Gravalos C, Salut A, Garcia-Giron C, Garcia-Carbonero R, Leon AI, Sevilla I, et al. A randomized phase II study to compare oxaliplatin plus 5-fluorouracil and leucovorin (FOLFOX4) versus oxaliplatin plus raltitrexed (TOMOX) as first-line chemotherapy for advanced colorectal cancer. Clin Transl Oncol. 2012;14:606-12.

26. Cascinu S, Graziano F, Ferrau F, Catalano V, Massacesi C, Santini D, et al. Raltitrexed plus oxaliplatin (TOMOX) as first-line chemotherapy for metastatic colorectal cancer. A phase II study of the Italian Group for the Study of Gastrointestinal Tract Carcinomas (GISCAD). Ann Oncol. 2002;13:716-20.

27. Seitz JF, Bennouna J, Paillot B, Gamelin E, Francois E, Conroy $\mathrm{T}$, et al. Multicenter non-randomized phase II study of raltitrexed (Tomudex) and oxaliplatin in non-pretreated metastatic colorectal cancer patients. Ann Oncol. 2002;13:1072-9.

28. Santini D, Massacesi C, D’Angelillo RM, Marcucci F, Campisi C, Vincenzi B, et al. Raltitrexed plus weekly oxaliplatin as first-line chemotherapy in metastatic colorectal cancer: a multicenter nonrandomized phase II study. Med Oncol. 2004;21:59-66.

29. Liu Y, Wu W, Hong W, Sun X, Wu J, Huang Q. Raltitrexed-based chemotherapy for advanced colorectal cancer. Clin Res Hepatol Gastroenterol. 2014;38:219-25.

30. Guo JH, Zhang HY, Gao S, Zhang PJ, Li XT, Chen H, et al. Hepatic artery infusion with raltitrexed or 5-fluorouracil for colorectal cancer liver metastasis. World J Gastroenterol. 2017;23:1406-11.

31. de Takats PG, Kerr DJ, Poole CJ, Warren HW, McArdle CS. Hepatic arterial chemotherapy for metastatic colorectal carcinoma. Br J Cancer. 1994;69:372-8.

32. Cercek A, Boucher TM, Gluskin JS, Aguilo A, Chou JF, Connell LC, et al. Response rates of hepatic arterial infusion pump therapy in patients with metastatic colorectal cancer liver metastases refractory to all standard chemotherapies. J Surg Oncol. 2016;114:655-63.

33. Takahashi H, Akyuz M, Aksoy E, Karabulut K, Berber E. Local recurrence after laparoscopic radiofrequency ablation of malignant liver tumors: results of a contemporary series. J Surg Oncol. 2017;115:830-4.

34. Berber E, Siperstein A. Local recurrence after laparoscopic radiofrequency ablation of liver tumors: an analysis of 1032 tumors. Ann Surg Oncol. 2008;15:2757-64.

35. Avallone A, Di Gennaro E, Silvestro L, Iaffaioli VR, Budillon A. Targeting thymidylate synthase in colorectal cancer: critical re-evaluation and emerging therapeutic role of raltitrexed. Expert Opin Drug Saf. 2014;13:113-29. 